J. Geomag. Geoelectr., 43, Suppl., 223-231, 1991

\title{
On Spontaneous Reconnection in the Earth's Magnetotail
}

\author{
J. BÜCHNER \\ Central Institute for Astrophysics of the Academy of Sciences, Potsdam, 1591, G.D.R.
}

(Received September 13, 1990; Revised January 28, 1991)

\begin{abstract}
The release of energy stored in the Earth's magnetotail is a major feature of the coupling between solar wind and magnetosphere. Spontaneous reconnection due to tearing mode instability in the magnetotail provides an appropriate mechanism, explaining energy release, delays the response to changes in the interplanetary medium, non stationary features of the magnetospheric behaviour. Problems, however, exist in the theory of spontaneous reconnection. Here in this paper new theoretical results are discussed, which help to advance the understanding of collisionless nonstationary reconnection processes in the tail. It is discussed, how the stabilizing influence of adiabatic electrons can be canceled. Further it is shown that in addition to the instability of a shearless tail, usually discussed, another route to instability appears in the presence of a finite magnetic shear. Finally the transition to a nonlinear, explosive mode of instability is examined.
\end{abstract}

\section{Introduction}

After decades of intense work on substorms it appears that no unified picture of the magnetospheric activity exists. Every substorm seems to have its own story. A number of observations allow, however, to summarize roughly main features of the interaction between the solar wind and the magnetosphere. No doubt remained that the Earth's magnetotail is a huge energy reservoir, supplied by the solar wind and that the magnetospheric activity is closely connected with a partial release of the energy, previously stored in the tail. In order to predict the activity onset and the amount of released energy one needs to know the corresponding physical mechanism. Reconnection is an appropriate concept, providing a direct conversion of magnetic and current flow energy to particle acceleration and heating. Reconnection in the tail can be considered in two limits. The so called "driven" limit requires an externally forced plasma inflow into the tail, which controls the reconnection process. This is related with observations of a direct correlation between sudden enhancements of the solar wind input and magnetospheric activity. However, the release of tail energy can also occur spontaneously in the course of the tail evolution from one stable configuration to other, without enhanced external plasma inflows. An appropriate theoretical description of this process has to explain the stable evolution, which means the existence of a critical level, behind which the magnetotail becomes unstable. Theoretical approaches to spontaneous reconnection have been discussed extensively in the literature, e.g., by SCHINDLER (1974), GALEEV and ZELENYI (1976), CORONITI (1985a). Here in this paper new results are put forward, which shows spontaneous reconnection and tearing mode instability as a more and more appropriate candidate, explaining the activity of the Earth's magnetotail. 


\section{Linear Stage}

Isolated substorms are characterized by a sudden onset, without remarkable perturbations in the solar wind. The onset criterion and the characteristic growth time of a tearing mode instability are two main parameters, which are able to verify the applicability of this concept to the physics of the magnetotail. Two necessary conditions must be met to destabilize a tearing mode instability

(1) a dissipation process must act to dissipate the particle kinetic energy inside the diffusion region of the current sheet

and

(2) the current dissipation must be compensated by the energy, released due to current filamentation.

To verify the second condition one has to know the free energy, available due to the sheet tearing. It is appropriate to start with a definite magnetotail field model like

$$
\boldsymbol{B}=B_{0} \cdot \tanh (z / L) \cdot e_{x}+B_{\mathrm{s}} \cdot e_{y}+B_{\mathrm{n}} \cdot e_{z}
$$

Together with the current-and density distribution of a HARRIS (1962)—equilibrium this model provides a lowest order approach to an asymptotic two-dimensional current sheet equilibrium, derived by SCHINDLER (1975), if the spatial scales of interest do not exceed $L_{x} \cong$ $L \cdot B_{0} / B_{\mathrm{n}}$, the inhomogeneity scale along the $x$-axis. The threshold and the growth rate of instability can be found analyzing the evolution of a tearing mode perturbation

$$
f(x, z, t)=\tilde{f}(z) \cdot \exp \{-i \omega t+i k x\}
$$

in the sense of a WKB approach. For a perturbation (2) the reversible (= free) energy variation in the dissipationless outer part of the current sheet equals (cf. GALEEV, 1984).

$$
\delta W_{\mathrm{f}}=-\frac{1-k^{2} L^{2}}{k L+\delta} \cdot \frac{\left|\tilde{A}_{10}\right|^{2}}{8 \pi}=-S(k L) \cdot \frac{\left|\tilde{A}_{10}\right|^{2}}{8 \pi}
$$

where $\tilde{A}_{10}=\tilde{A}_{1}(z=0)$. The term $S(k L)$, with $k=2 \pi / \lambda$ and the wavelength of the mode $\lambda$, describes the energy gain due to a tearing of the current sheet into filaments, i.e., the variation of released free energy, reduced by the energy, spent for the build-up of the perturbed magnetic field $\left|\tilde{B}_{1}\right| 2 / 8 \pi$. $\delta$ denotes the half-thickness (normalized to $L$ ) of the diffusion layer, where the current dissipation takes place.

For a given finite resistivity $\eta=c^{2} / 4 \pi \sigma(\sigma=$ conductivity) a general compressible MHD-solution for weakly two dimensional, unsheared $\left(B_{\mathrm{s}}=0\right)$ taillike equilibria was obtained by OTTO (1990). He found that the resistive growth time $\tau=\gamma^{-1}$ does not depend on the $B_{\mathrm{n}}$-component and equals that of an unstable one-dimensional sheet with $B_{\mathrm{n}}=0=B_{\mathrm{s}}$, derived first by FURTH et al. (1963):

$$
\gamma r=\left[\frac{\Gamma(1 / 4) \cdot S(k L)}{\pi \cdot \Gamma(3 / 4)}\right] \cdot S^{-3 / 5} \cdot \frac{1}{t_{\mathrm{A}}},
$$


where $\tau_{\mathrm{A}}=L / v_{\mathrm{A}}$ is the Alfvén time scale, $v_{\mathrm{A}}=\mathrm{B}_{0} / \sqrt{4 \pi M_{\mathrm{i}} n}$ the Alfvén velocity, $S=\tau_{\mathrm{D}} / \tau_{\mathrm{A}}$ the magnetic Reynolds-number with the diffusion time scale $\tau_{D}=4 \pi \sigma L^{2} / c^{2}=L^{2} / \eta$. MHDreconnection solutions were also obtained by means of MHD simulations, restricting, e.g., the finite plasma resistivity to certain spatially limited region, cf., e.g. SCHOLER (1989) and references therin. MHD models depend on assumptions about a finite resistivity providing current dissipation in a collisionless diffusion region. CORONITI (1985b) and BÜCHNER et al. (1988) argued that it is very difficult to find a sufficient anomalous resistivity, i.e., to meet the threshold of an appropriate current instability in the tail.

The nonideal response of the sheet current to tearing perturbations can be provided, however, also by inertial effects like Landau damping or chaotic particle scattering. In fact the magnetization of the charged particles may break down in inhomogeneous magnetic fields like that of the magnetotail. An approximate quantitative criterion for this transition can be formulated for a curved and sheared field reversal (1), following BÜCHNER and ZELENYI (1986) as:

$$
\kappa=\kappa_{\mathrm{n}} \cdot\left[1+\left(\frac{\kappa_{\mathrm{s}}}{\kappa_{\mathrm{n}}}\right)^{2}\right]^{3 / 4} \cong 1 \quad \text { where } \quad \kappa_{\mathrm{n} ; \mathrm{s}}=\frac{B_{\mathrm{n} ; \mathrm{s}}}{\mathrm{B}_{0}} \sqrt{\frac{L}{\rho_{0}}}
$$

with $\rho_{0}=v / \Omega_{0}$ and $v$ the particles total velocity and $\Omega_{0}$ as the Larmor-frequency in the asymptotic field $B_{0}$. The particle behaviour in dependence on parameters $\kappa_{\mathrm{n}}$ and $\kappa_{\mathrm{s}}$ for electrons is shown in Fig. 1. As long as $B_{\mathrm{s}} / B_{0}<\sqrt{\rho_{0} / L}$, i.e., $\kappa_{\mathrm{s}}<1$, particle trajectories are nonadiabatic and chaotic until $\kappa \approx \kappa_{n} \cong 3$ (the dashed line). In the limit $\kappa \approx \kappa_{n}<1$ the particles bounce up and down across the neutral plane. BÜCHNER and ZELENYI (1989) have shown that in this limit a quasiadiabatic type of motion appears, which is intercepted by finite jumps of the quasi-invariant $I=(2 \pi)^{-1} \int v_{z} d z$ during separatrix traversals in the phase space. The oscillation amplitude in the neutral-plane-crossing segment of quasiadiabatic orbits can be estimated as $\Delta_{j}=\sqrt{\rho_{0 j} L}$, where $j=i, e$ denotes the type of particles with $\rho_{0 j}=$ $v_{t j} / \Omega_{0 j}$ and $v_{t j}$ is the thermal velocity, $\Omega_{0 j}=e B_{0} /\left(m_{j} c\right)$. A finite magnetic shear field $B_{\mathrm{s}}$ starts to change the orbits essentially beginning with $B_{s} / B_{0}>\sqrt{\rho_{0} / L}\left(\kappa_{\mathrm{s}}>1\right)$, i.e., when the particle's gyration radius in $B_{\mathrm{s}}$ becomes less than $\Delta_{j}$. Electrons with parameters corresponding to the inner solid curve $\kappa_{\mathrm{e}}=1$ in Fig. 1 are completely chaotic. Assuming temperature values $T_{\mathrm{e}}=1 \mathrm{keV}$ and $T_{\mathrm{i}}=5 \mathrm{keV}$ for magnetotail electrons and ions, respectively, one finds for particles with the thermal velocities $\kappa_{\mathrm{i}}=10 \kappa_{\mathrm{c}}$. The resulting curve $\kappa_{\mathrm{i}}=1$ is shown (in the $\kappa_{\mathrm{e}}$-scale) to the right of Fig. 1. If one applies typical magnetotail parameter values of about $B_{0}=20 \mathrm{nT}$ and $L=3000 \mathrm{~km}$ (at $20 R_{\mathrm{E}}$ ) the most probable $\kappa_{\mathrm{n}}$-parameter of thermal electrons is larger than 2 , closer to 5 , that of ions, respectively, 0.2-0.5. From the values of the shear field components, observed by FAIRFIELD (1979), TSURUTANI et al. (1984), MCCOMAS et al. (1986) and SERGEEV (1987), one finds $\kappa_{\mathrm{s}}$ values for electrons from 0 to 5 . Consequently, the thermal magnetotail ions bounce across the neutral plane on quasiadiabatic orbits. Thermal electrons behave for $\kappa_{\mathrm{s}}<1$ rather chaotically than regularly, for finite $B_{\mathrm{s}}$, corresponding to $\kappa_{\mathrm{s}}>1$ they become regular, drifting mainly along the current direction. 


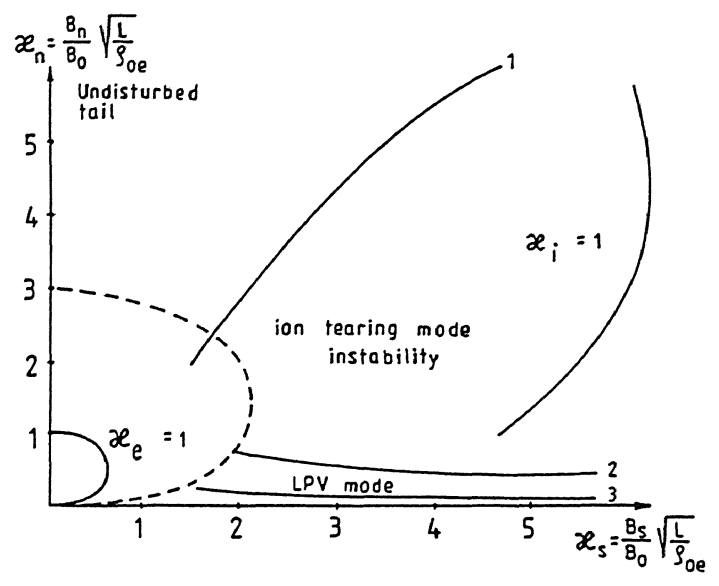

Fig. 1. Regions of regular (adiabatic, gyroscopic) and chaotic electron motion in a curved and sheared field reversal as far as routes to instability of the collisionless tearing mode shown in dependency on the $\kappa_{\mathrm{S}}$ and $\kappa_{\mathrm{n}}$ parameters for thermal electrons.

The energy variation caused by an interaction of a tearing mode perturbation with nonadiabatic particles, bouncing across the current sheet in a layer with the thickness $\Delta_{j}$ was expressed by BÜCHNER and ZELENYI (1988) as follows:

$$
\delta W_{j}=-i \omega L \cdot \Delta_{j} \cdot \tau_{j} \cdot \frac{\omega_{p j}^{2}}{c^{2}} \cdot \frac{\left|\tilde{A}_{10}\right|^{2}}{8 \pi}
$$

where $\omega_{p j}=\sqrt{4 \pi n e^{2} / m_{j}}$ is the plasma frequency of the specie ${ }_{j}$. In the case of Landau damping the characteristic time $\tau_{j}$ of energy exchange between the growing mode and particles equals, approximately, the travel time of a particle in wave potential well $\tau_{j}=$ $\left(k v_{t j}\right)^{-1}$. In a one-dimensional current sheet the Landau damping on electrons dominates $(j=$ $e$ ) and the collisionless electron tearing mode growth rate, first derived by COPPI $e t$ al. (1966), follows from Eqs. (3) and (6) as $(\gamma=-i \omega)$ :

$$
\gamma_{\mathrm{e}}=\frac{k v_{\mathrm{te}}}{\sqrt{\pi}} \cdot\left(\frac{\rho_{0 \mathrm{e}}}{L}\right)^{3 / 2} \cdot S(k L) \cdot\left(1+\frac{T_{\mathrm{i}}}{T_{\mathrm{e}}}\right)
$$

For realistic magnetotail parameters one finds that the linear electron tearing mode growth time $\gamma_{\mathrm{e}}{ }^{-1}$ is essentially to slow to explain substorm explosions. Also, in the Earth magnetotail the electron Landau damping is suppressed by electrons' magnetization in the finite normal magnetic field component. SCHINDLER (1974) suggested that the ion Landaudamping may cause a tearing mode instability, which will have the same form as (5), but with ion parameters instead of electron ones. To verify this hypothesis one has to compare the ion response $(6)(j=i)$ with that of the electrons, which can be found solving the trajectory integral: 


$$
\delta W_{\mathrm{e}}=\frac{\omega_{\mathrm{pe}}^{2}}{4 \pi c^{2} v_{\mathrm{te}}^{2}} \cdot \int_{-\infty}^{+\infty} d z d d v \cdot f_{\mathrm{oe}} \cdot v_{y} \cdot \tilde{A}_{1}^{*}\left[-i \omega \cdot \int_{-\infty}^{0} d \tau\left(v_{y} \cdot \tilde{A}_{1}-c \phi_{1}\right)\right]
$$

GALEeV and ZELENYI (1976), CORONTTI (1980), LEMBÉGE and PELLAT (1982) have shown that the reversible electron response to the tearing perturbation for adiabatic electrons in reversals with finite $B_{\mathrm{n}}$-field components but $B_{\mathrm{s}}=0$ exceeds the available free energy $W_{\mathrm{f}}(3)$.

Several mechanisms have been proposed, which might be able to cancel this electron stabilization:

- GOLDSTEIN and SCHINDLER (1978) discussed its release via field aligned currents into the ionosphere;

- GOLDSTEIN and SCHINDLER (1982) suggested that it may vanish on very long wavelengths;

- CORONITI (1980), ESARAY and MOLVIG (1987) proposed electron scattering by wave particle interaction;

- BÜCHNER and ZELENYI (1987) found that the electron chaotization cancels their stabilizing influence.

The first approach limits the growth time of the instability to the travel time of electrons from the neutral line region, which is supposed to form at $20 R_{\mathrm{E}}$, to tens of minutes. The second idea seems to apply to very large substorms, creating a magnetic island, comparable in size with the whole magnetospheric tail. The third needs a sufficient turbulence level. The chaotization of electrons (fourth proposal) takes place with diminishing $\kappa_{\mathrm{n}} \rightarrow 1$. As it was shown by HORTON and TAJIMA (1990), this lets decay the correlation along the trajectories. BÜCHNER and ZELENYI (1988) showed that in this case the characteristic time scale of the energy exchange between the mode and particles $\tau_{j}$ is the decorrelation time scale of about $\Omega_{\mathrm{n} j}{ }^{-1}$, which now determines the nonideal response and, via expression (6) in comparison with (3), the growth rate of the mode.

BÜCHNER et al. (1990) have derived another route to the tearing mode destabilization caused by a finite shear of the tail field $B_{s}$, as it was observed by FAIRFIELD (1979), TSURUTANI et al. (1984), MCCOMAS et al. (1986) and SERGEEV (1987). LAVAL et al. (1965) as far as DRAKE and LEE (1977) have previously investigated the influence of magnetic shear on current sheet stability, neglecting $B_{\mathrm{n}}$. Solving the integral (8) for the case of a finite shear field component $B_{\mathrm{s}}$ in the presence of $B_{\mathrm{n}}$, one finds that the main contribution from the electron interaction with the tearing mode is concentrated to a thin layer around the neutral plane. The half-thickness $\sqrt{\pi B_{\mathrm{n}} \cdot B_{\mathrm{s}} / k L\left(B_{\mathrm{n}}^{2}+B_{\mathrm{s}}^{2}\right)}$ of this layer defines the parameter $\delta$ in expression (3). In the solution of the integral (8) two principally different regimes can be found: In the first limit the electron bounce period $\tau_{\mathrm{be}}=4\left(L / v_{\mathrm{tc}}\right) \cdot \sqrt{B_{0}^{2}+B_{\mathrm{n}}^{2}+B_{\mathrm{s}}^{2}} / B_{\mathrm{n}}$ is much larger than the growth time of the instability $\tau=\gamma^{-1}\left(\gamma \cdot \tau_{\mathrm{bc}}>1\right)$. In this limit the guiding center drift of the electron during the interaction is only a short segment of its bounce orbit. In this limit one finds a shear-field electron tearing mode instability with the growth rate

$$
\gamma_{\mathrm{es}}=\frac{k v_{\mathrm{te}}}{\sqrt{\pi}} \cdot\left(\frac{\rho_{0 \mathrm{e}}}{L}\right)^{2} \cdot \frac{B_{0}}{B_{\mathrm{s}}} \cdot S(k L) \cdot\left(1+\frac{T_{\mathrm{i}}}{T_{\mathrm{e}}}\right)
$$


This mode is similar to that, discussed by LAVAL et al. (1965) or DRAKE and LEE (1977). In Fig. 1 the parameter range of this mode, called LPV, is located below line 3 .

More realistic is the case of a finite values of $B_{\mathrm{n}}$ and the shear field $B_{\mathrm{s}}$, i.e., the limit $\left.\gamma \cdot \tau_{\text {be }}<1\right)$. The complete dispersion relation can be obtained from the variational principle $\delta W_{\mathrm{f}}+\delta W_{\mathrm{e}}+\delta W_{\mathrm{i}}=0$. Solving integral (8) one has now to consider a bounce period smaller than the growth time of the mode. Together with expressions (3), (6) for ions and (8) for the electrons one obtains a dispersion relation, defining a border of stability, denoted by " 1 " in Fig. 1. The resulting border of stability is shown in Fig. 1 as a solid line, denoted " 1 ". The resulting growth rate is determined by the ion Landau damping with

$$
\gamma_{\mathrm{i}}=\frac{k v_{\mathrm{ti}}}{\sqrt{\pi}} \cdot\left(\frac{\rho_{0 \mathrm{i}}}{L}\right)^{2} \cdot \operatorname{Min}\left(1, \sqrt{\frac{\rho_{0 \mathrm{i}} \cdot B_{0}^{2}}{L \cdot\left(B_{\mathrm{s}}^{2}+B_{\mathrm{n}}^{2}\right)}}\right) \cdot S(k L) \cdot\left(1+\frac{T_{\mathrm{i}}}{T_{\mathrm{e}}}\right)
$$

As one can see, the electron stabilization can be overcome even for quite large $B_{\mathrm{n}}$, if only $B_{\mathrm{s}}$ is big enough.

The ion mode growth rate (10) exceeds that of the electron mode (9) considerably, i.e., a range exists, where $\gamma_{\mathrm{es}}<\tau_{\mathrm{be}}{ }^{-1}<\gamma_{\mathrm{i}}$. In this range both ion and electron contributions to the instability are comparable and an intermediate, mixed mode appears, whose parameter region is shown in Fig. 1 between lines 2 and 3.

Hence, after all, the linear phase of the tearing mode instability is determined by ion interaction with the mode. It leads to time scales of several minutes. This is the correct order for many substorms. A faster instability should arise after a new neutral line and a magnetic island have formed either in the course of the tearing mode growth. It also might be triggered by strong changes in the solar wind, like, e.g., northward turnings of the interplanetary magnetic field, discussed, e.g., by ROSTOKER (1983). In the following section we outline the transition from the linear stage to the nonlinear evolution of the instability.

\section{Nonlinear Evolution}

Numerical simulations of TERASAWA (1981) and ZELENYI et al. (1984) have shown that already formed magnetic islands grow explosively, i.e., faster than exponentially. It appears that in magnetic islands the electrons are adiabatically magnetized by the tearing perturbation $\tilde{B}_{1}$ (GALEEV et al., 1978). Hence the ions will carry the main part of the nonideal waveparticle interaction. A qualitatively different, essentially nonlinear behaviour of the tearing mode can be expected, if the half-width of magnetic island exceeds the extent of the main region of the ion interaction with the mode $\Delta_{j}$. One obtains the critical strength of the

perturbation $\tilde{B}_{1}\left(t_{0}\right)=k \cdot \rho_{0 \mathrm{i}} \cdot B_{0}$. Near the reconnection $X$-line the ions become accelerated by the induced electric field $E_{y}$ ind $=-\gamma \cdot \delta A_{1}(t) / c$. Depending on whether they belong to the loss cone or not, after acceleration the ions are mirrored back to the reversal or leave it. Those ions, which leave, will take their kinetic energy away from the current sheet. This enhances the ion current flow by $j_{1}=n \cdot e \cdot \Delta v_{y}$, where the velocity gain $\Delta v_{y}=e E_{y}{ }^{\text {ind }} \Delta t / m$ can be estimated using $\Delta t \approx \Omega_{\mathrm{oi}}^{-1}{\sqrt{v_{\mathrm{ti}} \cdot k \cdot B_{1} / B_{0}}}^{-1}$. This determines the time dependence of the growing perturbation as: 


$$
\tilde{B}_{1}(t)=\frac{\tilde{B}_{1}\left(t_{0}\right)}{1-\left(t-t_{0}\right) / \tau_{\mathrm{e}}},
$$

where

$$
\frac{1}{\tau_{\mathrm{e}}}=\frac{\tilde{B}_{1}\left(t_{0}\right)}{B_{0}} \cdot S(k L) \cdot\left(\frac{\rho_{0 \mathrm{i}}}{L}\right)^{3 / 2} \cdot\left(1+\frac{T_{\mathrm{e}}}{T_{\mathrm{i}}}\right) \cdot \Omega_{0 \mathrm{i}} .
$$

The time dependency (11a) describes an explosive tearing mode growth on a time scale $\tau_{\mathrm{e}}$, which for $t=t_{0}$, matches with the time scale of the linear ion tearing mode $\gamma_{\mathrm{i}}^{-1}$.

With the development of kinetic particle codes in two spatial dimensions numerical experiments become available, which could verify the correctness of the models of spontaneous reconnection and its nonlinear evolution. Even the most advanced attempts, however, still do not reach the necessary resolution, to investigate the ion-electron charge separation effects. So PRITCHETT et al. (1989) applied an explicit particle code to a twodimensional initial equilibrium state, considering the ions as particles and the electrons as a massless neutralizing background. Although they could not verify the charge separation effects, stabilizing the mode, they found a nonlinearly triggered instability for large initial perturbations. HEWETT et al. (1988) used the implicit code "AVANTI" to consider both species, ions and electrons, kinetically. However, they also did not resolve the full electron time scale. This was attempted by ZWINGMANN et al. (1990) with the code "VENUS", but with mass ratios $M_{\mathrm{i}} / m_{\mathrm{e}}$ still to small to reach the resolution, necessary for an investigation of charge separation effects.

\section{Conclusions}

Theoretical investigations approach more and more the solution of the key problems of spontaneous reconnection in the Earth's magnetotail, i.e., that of collisionless dissipation and destabilization of a tearing mode instability. There is now no doubt that the magnetotail energy storage may become disrupted even without strong solar wind plasma inflow, either due to strong external perturbation, or to a spontaneous reconnection instability. In unsheared tail fields the stabilizing influence of adiabatic electrons can be canceled either due to electron scattering in wave fields or by a transition of the electron motion to deterministic chaos, if $\kappa_{\mathrm{e}} \rightarrow 1$ in the course of the energy accumulation process. In sheared magnetotail fields the instability may take place even before the electrons become scattered, because their charge separation can be canceled by their current aligned motion. The resulting sheared field tearing mode instability is able to create, instead of two-dimensional plasmoids, magnetic flux ropes, which well explain corresponding satellite observations of SIBECK et al. (1984), SCHOLER et al. (1985), ELPHIC et al. (1986), SIBECK (1989). If the onset of magnetospheric activity in unsheared fields must be expected about 15-45 minutes after the beginning of an enhanced energy storage in the magnetotail (the period necessary to compress the sheet) the appearance of a shear field can cause an immediate energy release. The time scale of the mode growth is determined on its linear stage by the interaction between the ions and the unstable mode lasting several minutes. The wavelength of the most unstable spontaneous reconnection mode is $\lambda \approx 10 \cdot L$. A nonlinear tearing mode instability, however, may follow the linear one 
or may be triggered directly by a strong perturbation of the tail. In both cases an explosive expansion of magnetic islands takes place. The onset criteria and time scales, achieved by these reconnection processes, agree with observations. However, more detailed investigations can now be carried out, looking directly for the predicted observable features. Hopefully, in the near future the development of fully kinetic codes will provide a tool to verify the theoretical findings, discussed here, selfconsistently, including a simulation of the nonlinear evolution of the mode and the spectra of accelerated particles as another directly observable feature.

The author would like to express his gratitude to Prof. G. Haerendel, the convenor of the STEP meeting for valuable comments, to Dr. L. M. Zelenyi from the Moscow Space Research Institute, Prof. K. Schindler and Dr. A. Otto in Bochum, to Dr. M. Scholer, Munich and to the collegues from the University of California at Los Angeles, especially to Prof. M. AshourAbdalla, Prof. F. C. Coroniti, Prof. C. Kennel and Prof. R. Pellat for many exciting discussions of the problem of spontaneous reconnection.

\section{REFERENCES}

BÜCHNER, J. and L. M. ZelenYi, Deterministic chaos in the dynamics of charged particles near a magnetic field reversal, Physics Letters A, 118, 395, 1986.

BUCHNER, J. and L. M. ZELENYI, Chaotization of the electron motion as the cause of an internal magnetotail instability and substorm onset, J. Geophys. Res., 92, 13456, 1987.

BUCHNer, J. and L. M. ZelenYI, Reconnection Instability, ESA SP-285, Vol. 2, pp. 21-28, 1988.

BÜChNer, J., C. V. MeISTER, and B. Nikutowski, Reconnection and Microturbulence, ESA SP-285, Vol. 2, $35-40,1988$.

BÜCHner, J. and L. M. ZelenYi, Adiabatic and chaotic charged particle motion in two-dimensional magnetic field reversals 1. Basic Theory, J. Geophys. Res., 93, 1989.

BÜCHNer, J. and L. M. ZELENYI, Shear field magnetotail instability and the creation of magnetic flux ropes, Geophys. Res. Lett., 1990, submitted.

Coppi, B., Laval, and R. Pellat, Dynamics of the geomagnetic tail, Phys. Rev. Lett., 16, 1207, 1966.

Coroniti, F. V., On the tearing mode in quasi-neutral sheets, J. Geophys. Res., 85, 6719, 1980.

CORONIT, F. V., Explosive tail reconnection: The growth and expansion phases of magnetospheric substorms, J. Geophys. Res., 90, 7427-7447, 1985a.

Coroniti, F. V., Space plasma turbulent dissipation: reality or myth?, Space Sci. Rev., 42, 399, $1985 \mathrm{~b}$.

Drake, Y. F. and Y. C. LeE, Kinetic theory of tearing instabilities, Phys. Fluids., 20, 1341, 1977.

Elphic, R. C., C. A. Cattell, K. Takahashi, S. J. Bame, C. T. Russell, ISEe-1 and 2 observations of magnetic flux ropes in the magnetotail: FTE's in the plasma sheet?, Geophys. Res. Lett., 13, 648, 1986.

Esarey, E. and K. Molvig, A turbulent mechanism for substorm onset in the Earth's magnetotail, Geophys. Res. Lett., 14(4)L, 367, 1987.

FAirfield, D. H., J. Geophys. Res., 84, 1950, 1979.

Furth, Kileen, and M. D. Rosenbluth, Finite resistivity instabilities of a sheet pinch, Phys. Fluids, $\mathbf{6}$, $459,1963$.

Galeev, A. A. and L. M. Zelenyi, Tearing instability in plasma configurations, Sov. J. Phys., JETP, 43, $1113,1976$.

Galeev, A. A., F. V. Coroniti, and M. Ashour-Abdalla, Explosive tearing mode reconnection in the magnetospheric tail, Geophys. Res. Lett., 5, 707, 1978.

Galeev, A. A., in Basic Plasma Physics, edited by A. A. Galeev and R. N. Sudan, Vol. 2, North HollandAmsterdam, p. 305, 1984.

Goldstein, H. and K. Schindler, On the role of the ionosphere in substorms: Generation of field aligned currents, J. Geophys. Res., 83, 2574, 1978.

Goldstein, H. and K. Schindler, Large scale collision free instability of two dimensional plasma sheets, Phys. Rev. Lett., 48, 1468, 1982. 
HARRIS, E. G., On a plasma sheath separating region of oppositely directed magnetic fields, Nuovo Cimento, 23, 115, 1962.

Hewett, D. W., G. E. Francis, and C. E. MAX Magnetic reconnection simulation using the 2.5 D electromagnetic direct implicit code "AVANTI", Phys. Rev. Lett., 61, 893, 1988 and ESA SP-285, Vol. 2, 21-28, 1988.

HoRTON, W. and T. TAJIMA, Decay of correlations and the collisionless conductivity in the geomagnetic tail, Geophys. Res. Lett., 17, 123-126, 1990.

Laval, G., R. Pellat, and M. Vuillemin, in Plasma Phys. and Controlled Nuclear Fusion Research (International Atomic Energy Agency, Vienna, 1965), Vol. 2, p. 259, 1965.

Lembége, B. and R. Pellat, Stability of a thick two-dimensional quasineutral sheet, Phys. Fluids, 25, 1995, 1982.

Mccomas, D. J., C. T. Russell, R. C. Elphic, and S. J. BAME, The near-Earth crosstail current sheet: Detailed ISEE 1 and 2 case studies, J. Geophys. Res., 91, 4287, 1986.

Oтto, A., Linear Analysis of the resistive instability of weakly two-dimensional taillike equilibria, Phys. Fluids, 1991 (submitted).

Pritchett, P. L., F. V. Coroniti, R. Pellat, and H. Karimabadi, Collisionless reconnection in a quasineutral sheet near marginal stability, Geophys. Res. Lett., 16, 1269-1272, 1989.

Rostoker, G., Triggering of expansive phase intensifications of magnetospheric substorms by northward turnings of the interplanetary magnetic field, J. Geophys. Res., 88, 6981, 1983.

SCHINDLER, K., A theory of the substorm mechanism, J. Geophys. Res., 79, 2803, 1974.

SChindler, K., Plasma and fields in the magnetospheric tail, Space Sci. Rev., 17, 589, 1975.

Scholer, M., B. Klecker, D. Hovestadt, G. Gloeckler, F. M. Ipavich, and A. B. Galvin, Energetic particle characteristics of magnetotail flux ropes, Geophys. Res. Lett., 12, 191-194, 1985.

SCHOLER, M., Undriven magnetic reconnection in an isolated current sheet, J. Geophys. Res., 94, 8805$8812,1989$.

SERGEEV, V. A., On the penetration of the $B_{y}$-component of the interplanetary magnetic field, Geomagnetism i Aeronomia, 27, 612-615, 1987, in russ., see also: Sov. J. Geomagnetism and Aeronomy, 1987.

Sibeck, D. G., G. L. Siscoe, L. A. Slavin, E. J. Smith, S. J. Bame, and F. L. Scarf, Magnetotail Flux Ropes, Geophys. Res. Lett., 11, 1090, 1984.

SIBECK, D. G., Evidence for flux ropes in the Earth's magnetotail, in Physics of magnetic flux ropes, Geophysical Monograph 58, Proceedings of the conference, held on Bermudas, 1989, edited by C. T. Russell, E. R. Priest, L. C. LEE, p. 637, 1989.

TERASAWA, T., Numerical study of explosive tearing mode instability in one-component plasma, $J$. Geophys. Res., 86, 9007, 1981.

Tsurutani, B. T., D. E. Jones, R. P. Lepping, E. J. Smith, and D. G. Sibeck, The relationship between the IMF $B_{y}$ and distant tail (150-238 $R_{\mathrm{E}}$ ) lobe and plasmasheet $B_{y}$ fields, Geophys. Res. Lett., 11, 1082-1085, 1984.

Zelenyi, L. M., A. S. Lipatov, D. G. Lominadze, and A. L. Taktakishvili, The dynamics of energetic proton bursts in the course of magnetic field topology reconstructions in the Earth's magnetotail, Planet. Space Sci., 32, 313, 1984.

Zwingmann, W., J. Wallace, K. Schindler, and J. Birn, Particle simulation of magnetic reconnection in the magnetotail configuration, J. Geophys. Res., 95, 1990. 